\title{
EFECTO DEL AUMENTO DE JUGADORES EXTRANJEROS EN LA LIGA URUGUAYA DE BASKETBALL
}

\section{EFFECT OF INCREASE IN FOREIGN PLAYERS IN THE URUGUAYAN BASKETBALL LEAGUE}

Recibido el 20 de febrero de 2021 / Aceptado el 11 de junio de 2021 / DOI: 10.24310/riccafd.2021.v10i2.11952 Correspondencia: Sebastian Andres Corbo-Olivera. sebacorbo15@gmail.com

\section{Corbo-Olivera, S $^{\text {1BCDF}}$; González-Ramírez, A. ${ }^{2 A C D F}$}

${ }^{1}$ Corbo-Olivera, S. Instituto Superior de Educación Física, Universidad de la República, Uruguay, sebacorbo15@gmail.com

${ }^{2}$ González-Ramírez, A. Instituto Superior de Educación Física, Universidad de la República, Uruguay, andres.gonz.ramirez@gmail.com

Responsabilidades

${ }^{A}$ Diseño de la investigación. ${ }^{B}$ Recolector de datos. ${ }^{C}$ Redactor del trabajo. ${ }^{D}$ Tratamiento estadístico. ${ }^{\mathrm{E}} \mathrm{Apoyo}$ económico. Fldea original y coordinador de toda la investigación

\section{RESUMEN}

En la Liga Uruguaya de Basketball (LUB) se produjo un cambio de regla que permitió incorporar un tercer jugador extranjero a los equipos en la temporada 2018/19. Objetivo: analizar los rendimientos de los jugadores masculinos extranjeros y nacionales de la LUB durante las temporadas $2017 / 18$ y 2018/19. Metodología: se realizó un análisis descriptivo con estadísticos de tendencia central, también pruebas paramétricas y no paramétricas en una muestra conformada por 964 registros estadísticos, de 111 jugadores masculinos participantes, en un total de 67 partidos. Resultados: los extranjeros dominaron en la mayoría de los estadísticos seleccionados, los jugadores nacionales tiran más de 3 puntos con un porcentaje de acierto mayor, también reciben menos tapas y pierden menos pelotas. A su vez, los extranjeros jugaron más minutos promedio. Conclusión: el cambio de regla implementado en la LUB ha modificado algunos indicadores del rendimiento tanto de jugadores extranjeros como nacionales. 


\section{PALABRAS CLAVE}

análisis de rendimiento, estadísticas de juego, básquetbol, jugadores nacionales, jugadores extranjeros.

\section{ABSTRACT}

In the Uruguayan Basketball league occurred a change in the rules that permited add a third foreign player to the teams in the 2018/19 season. Objective: analyze the performance of foreign and national masculine players of the LUB during the season 2017/18 and 2018/19. Methodology: it has been made a descriptive analysis with central tendency statistics, also parametric and no parametric tests in a shows made by 964 statistics records, of 111 participating masculine players, in a total of 67 games. Results: the foreign players dominated on the most of selected statistics, national players take more shots of 3 point with high success rate, also get less blocks and lose less balls. At the same, foreign players play more average minutes. Conclusion: rule change implemented in the LUB has modified some of performance indicators both for foreign for national players.

\section{KEY WORDS}

performance analysis, game related statistics, basketball, national players, foreign players.

\section{INTRODUCCIÓN}

La Liga Uruguaya de Basketball (LUB) es la categoría máxima de competencia dentro de la Federación Uruguaya de Basketball (FUBB). En el año 2017, tras una votación de los clubes, se confirmó un cambio reglamentario que permitió incorporar un tercer jugador extranjero en los equipos ${ }^{1}$. Los cambios de reglamentos pueden modificar la forma de juego en el deporte, Montero, Vila y Longarela ${ }^{2}$ evidenciaron que luego del cambio de la distancia de la línea de 3 puntos a 6,75 metros, se modificó la cantidad de tiros tomados, aumentaron los tiros de 3 puntos disminuyendo los tiros de campos de larga distancia y los porcentajes de aciertos. Además, el ingreso de extranjeros a ligas nacionales es un tema estudiado a nivel mundial luego del caso Bosman, ya que existió un incremento en los traspasos en el deporte. Se removieron las restricciones para la movilidad de los atletas lo que causó un incremento en el porcentaje de jugadores extranjeros en ligas europeas, ${ }^{4,5}$. 
En un informe presentado por FIBA ${ }^{6}$ en 2020 se mostró el alto número de jugadores extranjeros en las ligas nacionales. De 16 ligas estudiadas, en 6 predominan los extranjeros, destacándose España con el porcentaje más alto, seguido en América por Argentina y Brasil. También, Estados Unidos forma parte de este proceso, con un incremento de jugadores extranjeros en sus diversas ligas (NCAA, WNBA, NBA) en los tres últimos años. Este informe sugiere que el predomino de jugadores extranjeros podría estar limitando el desarrollo deportivo de los jugadores nacionales.

Sin embargo, los rendimientos de estos países en competencias internacionales son destacables. Estados Unidos se colocó en el primer puesto del ranking mundial, España en el segundo, Argentina se posicionó en el cuarto puesto, mientras que Brasil se encuentra en el décimo ${ }^{7}$. Además, a favor de los extranjeros en las ligas, Baur y Lehmann ${ }^{3}$ mencionan que las selecciones nacionales que tienen más jugadores en el exterior mostraron mejores rendimientos internacionales, y a su vez los jugadores nacionales mejoraron sus habilidades técnico-tácticas. Álvarez et al. ${ }^{5}$ exponen que si bien pueden existir argumentos acerca de efectos negativos del talento extranjero (por ejemplo, retraso en los jugadores locales en asegurarse un lugar en el equipo principal), la evidencia demuestra que diferentes aspectos negativos en el básquet europeo fueron paliados por la influencia de los extranjeros. En la misma línea, Meletakos et al. ${ }^{4}$ confirmaron que la presencia de jugadores extranjeros en la liga local griega ha mejorado el rendimiento de la selección nacional en la Eurobasket.

Por contraparte, para el análisis de rendimiento son buenos indicadores las estadísticas individuales y colectivas utilizadas en las competiciones ${ }^{8}$. Existe numerosa bibliografía en donde se demuestra cómo las variables estadísticas diferencian a ganadores de perdedores. Ibañez et al. ${ }^{9}$ encontraron que en la liga U20 de España, la variable más relevante fueron los tiros de campo de 3 puntos; Gómez et al. ${ }^{10}$ mostraron que, en el básquetbol profesional de España (ACB) y en los partidos con resultado equilibrado (menores o iguales a 12 puntos), los rebotes defensivos fueron determinantes, mientras que en los no equilibrados existió un mayor acierto de 2 puntos, de rebotes defensivos y de asistencias. También Gómez, Lorenzo, y Barakat ${ }^{11}$ encontraron en la misma liga que los rebotes defensivos y las asistencias fueron las variables más poderosas, los equipos vencedores que jugaron de local fueron más agresivos en defensa, mientras que los visitantes ganadores tiraron mejor de 2 puntos, erraron de 3 puntos, hicieron más asistencias y tomaron más rebotes defensivos. Adicionalmente García et al. ${ }^{12}$ identificaron que en la ACB los equipos ganadores en sus canchas destacaron en tiros de 2 puntos hechos, tapones (tapas) realizadas, mates (hundidas), rebotes defensivos y asistencias. Ibañez et al. ${ }^{13}$ 
analizaron otra liga de España (LEB1) y encontraron que las variables que definieron los mejores y peores equipos fueron las asistencias, robos y tapas realizadas, mientras que lo que definió a los ganadores fueron los rebotes defensivos y los tiros de campo.

Asimismo, existe bibliografía en donde se estudió otras ligas del mundo. Mandarme ${ }^{14}$ expuso que tanto en Asia como en Europa la variable que diferenció a los ganadores en los juegos equilibrados fueron los rebotes defensivos, mientras que en los desequilibrados fueron los tiros 2 puntos convertidos, rebotes defensivos y asistencias. El mismo autor realizó otro estudio con competencias intercontinentales femeninas U18, en donde expuso que Asia se diferenció de las demás ligas por el número de posesiones, los rebotes defensivos, las asistencias y las faltas recibidas. Por su parte, África se diferenció de Europa por el número de posesiones, los aciertos en tiro de 3 puntos, los tiros libres errados y los robos, mientras que la diferencia con América radica en el número de posesiones, tiros de 2 puntos errados, tiros de 3 puntos anotados, acierto en tiro libres y asistencias ${ }^{15}$. Almas ${ }^{16}$ estudió la NBB de Brasil y encontró que las estadísticas relevantes en las victorias para partidos equilibrados y no equilibrados en temporada regular y partidos equilibrados en playoff fueron los tiros de 3 puntos convertidos y los rebotes defensivos. Por último, Paulauskas et al. ${ }^{17}$ compararon el rendimiento de los jugadores de Europa y de la NBA y revelaron que las diferencias estuvieron relacionadas con la influencia del tamaño corporal.

Por otra parte, Mikić et al. ${ }^{18}$ afirmaron que los rebotes defensivos fueron la única estadística que diferenció a los ganadores de perdedores, y además destacaron la importancia de la preparación para la presión defensiva. Ortega-Toro et al. ${ }^{19}$ afirmaron que los ganadores alternan más defensas individuales que zonales, que las diferencias en el marcador ocurrieron en los primeros y terceros cuartos, y que en el último cuarto los rebotes defensivos fueron mayores en los equipos perdedores. En Uruguay, González-Ramírez y Fernández ${ }^{20}$ afirmaron que el rebote defensivo no fue diferenciador entre equipos ganadores y perdedores en playoff, además el sistema defensivo más utilizado fue la defensa individual mostrando un nivel elevado de eficacia.

También se puede encontrar estudios relacionados a las estadísticas y las fases de juego. Dogan y Ersoz ${ }^{21}$ encontraron que en la Euroliga el porcentaje de acierto de 3 puntos fue considerado como la variable esencial en cada ronda; García et al. ${ }^{22}$ expusieron que los equipos ganadores en la temporada regular dominaron en asistencias, rebotes defensivos, y tiros de 2 y 3 puntos convertidos, mientras que en playoff solo en rebotes defensivos. Por último, Mateus et al. ${ }^{23}$ encontraron 4 perfiles de rendimiento diferentes en playoff de la NBA. 
Por otra parte, existen publicaciones referidas a las diferencias en el rendimiento entre hombres y mujeres. García, Ibañez y Feu ${ }^{24}$ demostraron que los hombres presentaron mayores capacidades físicas, mayor capacidad para lanzar a distancia, intensidad defensiva y número de asistencias. Madarme ${ }^{25}$ demostró que los tiros libres, pero no las asistencias discriminaron los ganadores en U17, los tiros de 2 puntos acertados discriminaron los ganadores solo en mujeres y los tiros de 3 puntos acertados en mujeres fueron significativamente más bajas que en hombres.

En cuanto a estudios de extranjeros y nacionales en las diferentes ligas, Guimarães et al. ${ }^{26}$ demostraron que en la Portuguese Basketball League (LPB), los extranjeros fueron superiores en la mayoría de las variables con excepción en los intentos de triples, asistencias y balones recuperados donde dominaron los nacionales. Gasperi et al. ${ }^{27}$ evidenciaron que las extranjeras exhibieron mayores valores en tiempo jugado, en tiros libres y tiros de dos puntos en la mayoría de las posiciones, en asistencias para armadoras y aleras, en rebotes defensivos por partes de las pívots y en robos por parte de las aleras. Por último, Manasses ${ }^{28}$ demostró que los extranjeros jugaron más minutos promedio que los nacionales, dominaron en tiros de 3 puntos y libres, así como también en pérdidas, en rebotes ofensivos y defensivos y tuvieron una mayor eficacia.

Por lo expuesto anteriormente se plantea en este estudio analizar los rendimientos de los jugadores masculinos extranjeros y nacionales de la Liga Uruguaya de Basketball durante las temporadas 2017/18 y 2018/19.

\section{MATERIAL Y MÉTODOS}

Para el cumplimiento del objetivo planteado se realizó un estudio descriptivo de tipo cuantitativo.

\section{Muestra}

La muestra constó de 964 registros de 108 jugadores masculinos participantes en un total de 68 partidos de los playoffs de la Liga Uruguaya Basketball en las temporadas 2017/2018 y 2018/2019. Se excluyeron aquellos jugadores que jugaron menos de 10 minutos por partido ${ }^{29}$. 
Tabla 1. Número de partidos, equipos e instancias de competición de las series de playoffs analizadas.

\begin{tabular}{|c|c|c|c|}
\hline Temporada & Fase torneo & Partido & $\begin{array}{l}\text { Partidos } \\
\text { jugados }\end{array}$ \\
\hline $2017 / 2018$ & Cuartos de final & Aguada vs Urunday Universitario & 3 \\
\hline $2017 / 2018$ & Cuartos de final & Malvin vs Hebraica Macabi & 5 \\
\hline $2017 / 2018$ & Cuartos de final & Goes vs Defensor Sporting & 4 \\
\hline $2017 / 2018$ & Cuartos de final & Welcome vs Nacional & 5 \\
\hline $2017 / 2018$ & Semifinales & Aguada vs Nacional & 5 \\
\hline $2017 / 2018$ & Semifinales & Malvin vs Defensor & 4 \\
\hline $2017 / 2018$ & Finales & Aguada vs Malvin & 7 \\
\hline $2018 / 2019$ & Cuartos de final & Aguada vs Defensor Sporting & 4 \\
\hline $2018 / 2019$ & Cuartos de final & Trouville vs Urunday Universitario & 5 \\
\hline $2018 / 2019$ & Cuartos de final & Nacional vs Olimpia & 5 \\
\hline $2018 / 2019$ & Cuartos de final & Malvin vs Biguá & 5 \\
\hline $2018 / 2019$ & Semifinales & Aguada vs Nacional & 5 \\
\hline $2018 / 2019$ & Semifinales & Malvin vs Urunday Universitario & 4 \\
\hline $2018 / 2019$ & Finales & Aguada vs Malvin & 7 \\
\hline
\end{tabular}

\section{Procedimiento}

Para la recolección de datos se recurrió a las estadísticas proporcionadas por FIBA en su página web https://www. fibalivestats. com/. Se seleccionaron los datos de los equipos clasificados para los playoffs de las LUB 2017/2018 y 2018/2019, fase donde se encuentran los 8 mejores equipos de la temporada regular ${ }^{21,22}$. En una planilla de cálculo Excel se transcribieron los datos de todos los jugadores que conformaron los equipos en cada partido. Posteriormente se realizó su tratamiento estadístico.

\section{Variables}

Las variables seleccionadas para el análisis fueron los minutos jugados (Min), los puntos convertidos (PTS), tiros acertados de 2 puntos (T2A), tiros fallados de 2 puntos (T2F), porcentaje de acierto de 2 puntos (T2\%), tiros acertados de 3 puntos (T3A), tiros fallados de 3 puntos 
(T3F), porcentaje de acierto de 3 puntos (T3\%), tiros acertados de 1 puntos (T1A), tiros fallados de 1 puntos (T1F), porcentaje de acierto de 1 punto (T1\%), rebotes ofensivos (RO), rebotes defensivos (RD), total de rebotes (REB), asistencias (AS), perdidas (PER), robos de balón (ST), tapas realizada (BLQ), tapas recibidas (BR), faltas personales (FP), faltas recibidas (FR).

\section{Análisis estadístico}

Se realizó un análisis de tipo descriptivo con estadísticos de tendencia central, también pruebas paramétricas y no paramétricas para comparar los resultados de los grupos en cuestión. Se calcularon las medias (M) y los desvíos estándar (SD), así como se procedió a utilizar la prueba paramétrica de Kolmogorov Smirnov para determinar la normalidad. Este test demostró que la muestra tenía una distribución libre, por lo que se utilizó la prueba $U$ no paramétrica de Mann Withney con el fin de determinar las diferencias significativas en los grupos y variables seleccionadas, con un intervalo de confianza del 95\%. Se utilizó el programa JASP $(0.14 .0 .0)$ para el tratamiento de los datos.

\section{RESULTADOS}

Se exponen los resultados de las variables seleccionadas en relación a la temporada de competencia en donde se destacaron los PTS, T2\% y $\mathrm{T} 1 \%$. Sin embargo, solamente se observaron diferencias significativas en PTS, T2A, AS y PER (tabla 2).

Tabla 2. Variables en función a las temporadas.

\begin{tabular}{|l|c|c|c|}
\hline Variable & 2018 & 2019 & $P$ \\
\hline PTS & $11,27 \pm 7,29$ & $10,08 \pm 7,14$ & $0,01^{*}$ \\
\hline T2A & $3,07 \pm 2,52$ & $2,67 \pm 2,43$ & $0,01^{*}$ \\
\hline T2F & $2,76 \pm 2,14$ & $2,74 \pm 2,27$ & 0,54 \\
\hline T2\% & $47,90 \pm 27,78$ & $44,85 \pm 29,96$ & 0,08 \\
\hline T3A & $1,10 \pm 1,29$ & $0,94 \pm 1,18$ & 0,07 \\
\hline T3F & $2,18 \pm 1,93$ & $1,90 \pm 1,69$ & 0,06 \\
\hline T3\% & $24,74 \pm 27,14$ & $23,22 \pm 26,57$ & 0,33 \\
\hline T1A & $1,83 \pm 2,01$ & $1,95 \pm 2,24$ & 0,83 \\
\hline T1F & $0,78 \pm 1,11$ & $0,81 \pm 1,27$ & 0,83 \\
\hline
\end{tabular}




\begin{tabular}{|l|c|c|c|}
\hline Variable & 2018 & 2019 & P \\
\hline T1\% & $50,64 \pm 41,31$ & $47,90 \pm 41,20$ & 0,29 \\
\hline RO & $1,32 \pm 1,61$ & $1,17 \pm 1,44$ & 0,29 \\
\hline RD & $3,25 \pm 2,74$ & $3,18 \pm 2,74$ & 0,55 \\
\hline REB & $4,57 \pm 3,75$ & $4,35 \pm 3,62$ & 0,29 \\
\hline AS & $2,44 \pm 1,97$ & $2,01 \pm 1,83$ & $<, 01^{* *}$ \\
\hline PER & $1,73 \pm 1,50$ & $1,50 \pm 1,37$ & $0,02^{*}$ \\
\hline ST & $0,96 \pm 1,09$ & $0,82 \pm 0,99$ & 0,04 \\
\hline BLQ & $0,33 \pm 0,68$ & $0,35 \pm 0,72$ & 0,75 \\
\hline BR & $0,34 \pm 0,59$ & $0,35 \pm 0,65$ & 0,84 \\
\hline FP & $2,69 \pm 1,43$ & $2,57 \pm 1,34$ & 0,17 \\
\hline FR & $2,79 \pm 2,16$ & $2,60 \pm 2,16$ & 0,13 \\
\hline
\end{tabular}

Los resultados en función de los jugadores nacionales y extranjeros mostraron que en la mayoría de las variables se destacaron los extranjeros, a excepción de T3A, T3F y T3\%, en donde los nacionales parecieron mostrar mejores porcentajes de acierto. Se pudo observar diferencias significativas en la mayoría de las variables, salvo en AS, ST y BR (tabla 3).

Tabla 3. Rendimiento de los jugadores extranjeros y nacionales.

\begin{tabular}{|c|c|c|c|}
\hline Variable & Extranjeros & Nacionales & $p$ \\
\hline PTS & $14,62 \pm 7,32$ & $8,31 \pm 6,09$ & $<0,01^{* *}$ \\
\hline $\mathrm{T} 2 \mathrm{~A}$ & $4,60 \pm 2,51$ & $1,83 \pm 1,80$ & $<0,01^{* *}$ \\
\hline $\mathrm{T} 2 \mathrm{~F}$ & $4,18 \pm 2,31$ & $1,92 \pm 1,65$ & $<0,01^{* *}$ \\
\hline $\mathrm{T} 2 \%$ & $51,55 \pm 20,70$ & $43,17 \pm 32,51$ & $<0,01^{* *}$ \\
\hline T3A & $0,88 \pm 1,29$ & $1,08 \pm 1,19$ & $<0,01^{* *}$ \\
\hline T3F & $1,72 \pm 1,96$ & $2,20 \pm 1,69$ & $<0,01^{* *}$ \\
\hline $\mathrm{T} 3 \%$ & $18,90 \pm 25,03$ & $26,83 \pm 27,42$ & $<0,01^{* *}$ \\
\hline $\mathrm{T} 1 \mathrm{~A}$ & $2,76 \pm 2,40$ & $1,40 \pm 1,79$ & $<0,01^{* *}$ \\
\hline $\mathrm{T} 1 \mathrm{~F}$ & $1,24 \pm 1,48$ & $0,53 \pm 0,91$ & $<0,01^{* *}$ \\
\hline $\mathrm{T} 1 \%$ & $59,40 \pm 35,96$ & $43,21 \pm 42,96$ & $<0,01^{* *}$ \\
\hline RO & $1,90 \pm 1,72$ & $0,85 \pm 1,23$ & $<0,01^{* *}$ \\
\hline
\end{tabular}




\begin{tabular}{l|c|c|c}
\hline Variable & Extranjeros & Nacionales & P \\
\hline RD & $4,73 \pm 2,88$ & $2,33 \pm 2,22$ & $<0,01^{* *}$ \\
REB & $6,64 \pm 3,81$ & $3,18 \pm 2,93$ & $<0,01^{* *}$ \\
AS & $2,20 \pm 1,63$ & $2,20 \pm 2,05$ & 0,16 \\
PER & $1,97 \pm 1,44$ & $1,39 \pm 1,39$ & $<0,01^{* *}$ \\
ST & $0,98 \pm 1,13$ & $0,83 \pm 0,98$ & 0,13 \\
BLQ & $0,67 \pm 0,93$ & $0,15 \pm 0,42$ & $<0,01^{* *}$ \\
BR & $0,38 \pm 0,70$ & $0,32 \pm 0,58$ & 0,60 \\
FP & $2,84 \pm 1,40$ & $2,50 \pm 1,36$ & $<0,01^{* *}$ \\
FR & $3,83 \pm 2,32$ & $2,03 \pm 1,75$ & $<0,01^{* *}$ \\
\hline
\end{tabular}

En cuanto al rendimiento de extranjeros y nacionales por año, se observó una clara disminución del promedio en PTS, T2\%, T3\% y T1\% por parte de los nacionales, mientras que los extranjeros disminuyeron sus números en $\mathrm{T} 2 \%$ y $\mathrm{AS}$, pero con una mejora en T3\% y T1\%. Se observaron diferencias significativas de los nacionales en PTS, T2A, T2F, T3A, RO y REB mientras que de los extranjeros en T2A, AS, PER Y BR (tabla 4).

Tabla 4. Rendimiento de extranjeros y nacionales por año.

\begin{tabular}{|l|c|c|c|c|c|c|}
\multicolumn{7}{|c}{ Nacionales } \\
\hline Variable & 2018 & 2019 & $\mathbf{P}$ & 2018 & 2019 & $\mathbf{P}$ \\
\hline PTS & $9,43 \pm 6,76$ & $7,28 \pm 5,19$ & $<0,01^{* *}$ & $14,87 \pm 6,96$ & $14,43 \pm 7,58$ & 0,41 \\
T2A & $2,13 \pm 1,95$ & $1,56 \pm 1,60$ & $<0,01^{* *}$ & $4,91 \pm 2,49$ & $4,38 \pm 2,52$ & $0,04^{*}$ \\
T2F & $2,10 \pm 1,72$ & $1,76 \pm 1,58$ & $0,01^{*}$ & $4,08 \pm 2,28$ & $4,26 \pm 2,34$ & 0,42 \\
T2\% & $44,64 \pm 30,72$ & $41,83 \pm 34,06$ & 0,25 & $54,30 \pm 19,42$ & $49,55 \pm 21,41$ & 0,22 \\
T3A & $1,23 \pm 1,32$ & $0,95 \pm 1,04$ & $0,03^{*}$ & $0,85 \pm 1,19$ & $0,91 \pm 1,36$ & 0,95 \\
T3F & $2,32 \pm 1,78$ & $2,09 \pm 1,59$ & 0,16 & $1,89 \pm 2,17$ & $1,61 \pm 1,78$ & 0,45 \\
T3\% & $28,13 \pm 27,59$ & $25,63 \pm 27,25$ & 0,19 & $18,09 \pm 25,01$ & $19,48 \pm 25,09$ & 0,62 \\
T1A & $1,49 \pm 1,89$ & $1,31 \pm 1,69$ & 0,20 & $2,50 \pm 2,08$ & $2,95 \pm 2,60$ & 0,20 \\
T1F & $0,54 \pm 0,86$ & $0,53 \pm 0,96$ & 0,49 & $1,24 \pm 1,37$ & $1,24 \pm 1,55$ & 0,72 \\
T1\% & $46,82 \pm 43,40$ & $39,90 \pm 42,34$ & 0,05 & $58,13 \pm 35,84$ & $60,31 \pm 36,11$ & 0,51 \\
RO & $0,99 \pm 1,40$ & $0,73 \pm 1,05$ & $0,04^{*}$ & $1,97 \pm 1,80$ & $1,86 \pm 1,67$ & 0,70 \\
RD & $2,49 \pm 2,36$ & $2,18 \pm 2,08$ & 0,13 & $4,75 \pm 2,83$ & $4,72 \pm 2,92$ & 0,73 \\
REB & $3,48 \pm 3,24$ & $2,92 \pm 2,60$ & $0,03^{*}$ & $6,72 \pm 3,76$ & $6,58 \pm 3,86$ & 0,54 \\
AS & $2,34 \pm 2,09$ & $2,08 \pm 2,01$ & 0,14 & $2,63 \pm 1,71$ & $1,89 \pm 1,49$ & $<0,01^{* *}$
\end{tabular}




\begin{tabular}{l|c|c|c|c|c|c}
\multicolumn{7}{c}{ Nacionales } \\
\hline Variable & $\mathbf{2 0 1 8}$ & $\mathbf{2 0 1 9}$ & $\mathbf{P}$ & $\mathbf{2 0 1 8}$ & $\mathbf{2 0 1 9}$ & $\mathbf{P}$ \\
\hline PER & $1,50 \pm 1,48$ & $1,29 \pm 1,30$ & 0,10 & $2,17 \pm 1,44$ & $1,82 \pm 1,42$ & $0,02^{*}$ \\
ST & $0,91 \pm 1,04$ & $0,75 \pm 0,92$ & 0,06 & $1,06 \pm 1,18$ & $0,91 \pm 1,10$ & 0,27 \\
BLQ & $0,15 \pm 0,43$ & $0,15 \pm 0,41$ & 0,80 & $0,68 \pm 0,91$ & $0,66 \pm 0,95$ & 0,65 \\
BR & $0,37 \pm 0,61$ & $0,28 \pm 0,55$ & 0,06 & $0,28 \pm 0,56$ & $0,45 \pm 0,78$ & $0,04^{*}$ \\
FP & $2,58 \pm 1,39$ & $2,43 \pm 1,32$ & 0,16 & $2,91 \pm 1,49$ & $2,79 \pm 1,34$ & 0,36 \\
FR & $2,15 \pm 1,77$ & $1,91 \pm 1,72$ & 0,06 & $4,05 \pm 2,30$ & $3,67 \pm 2,33$ & 0,16 \\
\hline
\end{tabular}

Por último, se exponen los minutos jugados tanto para los jugadores nacionales como para los extranjeros en 2018 y 2019 (tabla 5).

Tabla 5. Minutos jugados por nacionales y extranjeros.

\begin{tabular}{l|c|c|c|c|} 
& \multicolumn{3}{c}{ Nacionales } & \multicolumn{2}{c}{ Extranjeros } \\
\hline & 2018 & 2019 & 2018 & 2019 \\
\hline $\mathrm{N}^{\circ}$ registros & 292 & 318 & 149 & 205 \\
\hline $\mathrm{N}^{\circ}$ partidos & 33 & 35 & 33 & 35 \\
Total minutos jugados & 7.980 & 7.515 & 4.832 & 6.211 \\
Promedio de tiempos por partido & $27: 20$ & $23: 38$ & $32: 26$ & $30: 18$ \\
\hline
\end{tabular}

\section{DISCUSIÓN}

A partir del objetivo de analizar los rendimientos de los jugadores masculinos extranjeros y nacionales de la LUB, se pudo observar que entre las temporadas 2017/18 y 2018/19 se vieron modificados los rendimientos de los jugadores en la mayoría de las variables de estudio. Las mayores diferencias se presentaron en PTS, T2A, AS y PER y están relacionadas con la fase ofensiva. Estos resultados mostraron que la variación en el reglamento podría haber afectado el desarrollo del juego ofensivo de los equipos, confirmando que un cambio en el reglamento influyó directamente en la forma de jugar ${ }^{2}$, al menos en playoff.

Por otra parte, comparando a los jugadores extranjeros y nacionales en las dos temporadas, se observaron mejores valores en aspectos como el tiro exterior, las pérdidas, las tapas y las faltas personales en jugadores nacionales. Estas variables están relacionadas a la creación de juego en ofensiva y son similares a los resultados de Guimarães et al. ${ }^{26}$ donde observaron que los nacionales lideraron también en las estadísticas relacionadas a la creación de situaciones para el tiro y la 
anotación de compañeros (lo que definen como asistencias). Esto podría estar indicando que el rol principal de los jugadores nacionales es de armadores del ataque.

Por su parte, los extranjeros dominaron en el resto de variables, pudiendo indicar que su rol fue central en relación a la anotación y la toma de rebotes. La diferencia en REB pudo tener relación con su función dentro de la cancha, generalmente los extranjeros son jugadores internos (pívots o ala-pívots) que por su tamaño físico suelen especializarse en la toma de los rebotes ${ }^{29}$. Además, de una temporada a la otra, recibieron más tapas $(0,38$ frente a 0,32$)$ y perdieron más pelotas $(1,97$ frente a $1,39)$, lo que podría indicar que la defensa se focaliza en su marca y, además, aumenta su número de decisiones en ataque.

Respecto a los minutos jugados, se observó que los extranjeros tuvieron mayor cantidad de minutos totales jugados tanto en $2017 / 18$ como en $2018 / 19$, en comparación con los nacionales, resultado similar al presentado por Gasperi et al. ${ }^{27}$. Además, los nacionales disminuyeron sus minutos totales de una liga a la otra mientras que los extranjeros los aumentaron. En cuanto a los minutos promedios de un año al otro se observó una disminución en ambos grupos. Sin embargo, los nacionales disminuyeron su promedio de minutos en un $15 \%$ mientras que los extranjeros en un 6\%. Esto podría deberse a la distribución de minutos con el cambio de regla, ya que el tiempo de los nacionales en pista pudo haber disminuido en beneficio del tercer jugador extranjero. Según Manasses ${ }^{28}$ el promedio de minutos de los extranjeros en el equipo de U-Mobitelco fue de 30:84, similar a los jugados por los extranjeros en la LUB 2018/19 (30:18), mientras que el de los nacionales fue de 20:43, promedio bastante menor al observado en la misma (23:38).

En relación al tiro a distancia, los resultados observados en la LUB fueron similares a los obtenidos en la $\mathrm{LPB}^{26}$. En ambos, los jugadores nacionales tiraron más de 3 puntos y tuvieron una mayor eficacia. Además, se observó un mayor porcentaje de acierto los jugadores nacionales en la LUB en comparación a los del equipo U-Mobitelco ${ }^{28}$, tanto para tiros de 3 puntos $(26,83 \%$ y $12.30 \%$ respectivamente) como para tiros de 2 puntos ( $43,17 \%$ y $19.24 \%$ respectivamente), no observando grandes diferencias entre los valores de los extranjeros.

En cuanto a la comparación de los minutos jugados con el rendimiento en cancha de una temporada a la otra, los resultados mostraron que los nacionales disminuyeron la cantidad de minutos acompañado de un descenso en todas las variables. Por su parte, los extranjeros aumentaron sus minutos, pero no así su rendimiento, muchas variables no presentaron cambios significativos, otras aumentaron (T2F, T3A, T3\%, T1A, T1\% y BR) y otras disminuyeron (T2A, T2\%, T3F, RO, RD, REB, AS, PER, ST, BLQ, FP 
y FR). Estos resultados mostraron que haber jugado más minutos en la LUB no necesariamente implicó un aumento del rendimiento, indicando que no se estableció una relación directa entre el rendimiento y los minutos jugados.

Por último, González-Ramírez y Fernánde $z^{20}$ afirmaron que la capacidad de anotación fue un factor que definió a ganadores de perdedores y, por lo tanto, es importante mejorar la eficacia de los lanzamientos para la mejora del básquetbol uruguayo. Se observó que, en la finalización de las secuencias defensivas, las variables predominantes fueron las anotaciones y los rebotes defensivos. El éxito de los sistemas defensivos se basó en bajar la cantidad de anotaciones, en los equipos ganadores lograron que en un $66 \%$ de los lanzamientos no se logre la anotación, frente a un $59 \%$ de los perdedores. La presencia de extranjeros en la LUB parece haber beneficiado el desarrollo del tiro a distancia, lo que podría estar favoreciendo a la mejora en la capacidad de anotación por parte de los jugadores nacionales. A su vez, tanto en Brasil ${ }^{16}$ como en la Euroliga ${ }^{21}$, los aciertos en los tiros de tres puntos fueron determinantes para las victorias de los equipos. Los nacionales de la LUB presentaron mejores resultados en este rubro, por lo que los resultados presentaron semejanzas al juego internacional.

\section{CONCLUSIONES}

El cambio de regla parece haber modificado el rendimiento de los jugadores nacionales y extranjeros en los playoffs de la LUB. En este sentido, los jugadores extranjeros dominaron en la mayoría de las variables destacando su rol de reboteadores y anotadores, mientras que los jugadores nacionales mostraron dominio en las variables relacionadas al tiro de 3 puntos, así como también presentaron menor número de pérdidas, tapas y faltas personales, advirtiendo que su rol fue prioritariamente de armadores en el ataque. Por otra parte, los jugadores nacionales mostraron una disminución en la cantidad de tiros de 3 puntos realizados de una temporada a otra en comparación a los extranjeros, pero con una mayor eficacia que los extranjeros. Por último, ambos grupos de estudio disminuyeron sus promedios de minutos, pero el descenso de minutos totales jugados por los nacionales fue considerablemente mayor en comparación con los extranjeros.

\section{APLICACIONES PRÁCTICAS}

La información aportada por este estudio podrá ser de relevancia para los entrenadores de formativas de los equipos en Uruguay. Si los 
jugadores nacionales en LUB destacan en las estadísticas relacionadas a los tiros a distancia, el trabajo formativo deberá destacar los trabajos específicos de tiro de 3 puntos. Además, los resultados presentados sirven de insumo para realizar una captación de talento de jugadores nacionales con características antropométricas y técnico-tácticas similares a las de los extranjeros.

\section{BIBLIOGRAFÍA}

1. Reglamento de la Liga Uruguaya de Basketball. Montevideo: Federación Uruguaya de Basketball; 2018.

2. Montero, A; Vila, H; Longarela, B. Influence of change the distance of the 3-point line in basketball. J. Psychol. 2013; 22(1): 245-248.

3. Baur, DG; Lehmann, S. Does the Mobility of Football Players Influence the Success of the National Team?. IIIS [Internet]. 2007 [Consultado 2020 Dic 10]; No.217: 1-26. Disponible en: http://dx.doi.org/10.2139/ssrn.980936

4. Meletakos, P; Noutsos, K; Manasis, V; Bayios, L. The mediating effect of competitive balance on the relation between foreign players and performance of the Greek National Basketball Team. J. Phys. Educ. Sport Manag. 2014; 5(7): 72-80.

5. Alvarez, J; Forrest, D; Sanz, I; Tena, JD. Impact of importing foreign talent on performance levels co-wokers. Labour Econ. [Internet]. 2011[Consultado 2020 Dic 11]; 18(3): 287-296. Disponible en: https://doi. org $/ 10.1016 / j . l a b e c o .2010 .11 .003$

6. CIES Sport Observatory. International basketball migration report. Suiza: FIBA; 2020 pp. 1-81.

7. FIBA. FIBA WORLD RANKING, PRESENTADO POR NIKE. [Internet]. FIBA; 2020 Dic 09 [Consultado 2020 Dic 11]. Disponible en: http://www.fiba. basketball/es/rankingmen

8. Salmerón-Gómez, R; Haro-Gómez, S. Analizando horizontes sobre la medición del rendimiento y regularidad en el baloncesto profesional. Rev. Int. cienc. deporte. [Internet]. 2016 [Consultado 2020 Dic 12]; 12(45): 234-249. Disponible en: http://dx.doi.org/10.5232/ricyde2016.04502

9. Ibáñez, SJ; García, J; Feu, S; Lorenzo, A; Sampaio, J. Effects of consecutive basketball games on the game-related statistics that discriminate winner and losing teams. J Sports Sci Med. 2009; 8(3): 458-462.

10. Gómez, MA; Lorenzo, A; Sampaio, J; Ibáñez, SJ; Ortega, E. GameRelated Statistics tha Discriminate Winning and Losing Teams from the Spanish Men's Professional Basketball Teams. Coll. Antropol. 2008; 32(2): 451-456:

11. Gómez, MA; Lorenzo, A; Barakat, R. Differences in game relatedstatistics of basketball performance by game location for men's winning 
and losing teams. Percept. Mot. Ski. [Internet]. 2008. [Consul-tado 2020 Dic 22]; 106(1):43-50. Disponible en: doi:10.2466/PMS.106.1.43-50 Sport

12. García, J; Sáez, J; Ibáñez, SJ; Parejo, I; Cañadas, M. Home advantaje analysis in ACB league in season 2007-2008. J. Psychol. 2009; 18(1): 331-335.

13. Ibáñez, SJ; Sampaio, J; Feu, S; Lorenzo, A; Gómez, MA; Ortega, E. Basketball game-related statistics that discriminate between teams' seasonlong success. Eur J Sport Sci. [Internet]. 2008 [Consultado 2020 Dic 16]; 8(6): 369-372. Disponible en: http://dx.doi.org/10.1080/17461390802261470

14. Madarme, H. Game-Related Statistics Which Discriminate Between Winning and Losing Teams in Asian and European Men's Basketball Championships. Asian J Sports Med. [Internet]. 2017 [Consultado 2020 Dic 22]; 8(2): e42727. Disponible en: doi: 10.5812/asjsm.42727.

15. Madarme, H. Basketball Game-Related Statistics that Discriminate among Continental Championships for under-18 Women. Sports. [Internet]. 2018. [Consultado 2020 Dic 22]; 6(4): 114. Disponible en: https:// doi.org/10.3390/sports6040114

16. Almas, SP. Análise das estatísticas relacionadas ao jogo que discriminam as equipes vencedoras das perdedoras no basquetebol profissional brasileiro. Rev. bras. educ. fís. esporte. [Internet]. 2015. [Consultado 2020 Dic 22]; 29(4):551-58. Disponible en: https://doi.org/10.1590/180755092015000400551

17. Paulauskas, R; Masiulis, N; Vaquera, A; Figueira, B; Sampaio, J. Basketball Game-Related Statistics tha Discriminate Between European Players Competing in the NBA and in the Euroleague. J. Human Kinet. [Internet]. 2018 [Consultado 2020 Dic 16]; 65(1): 255-233. Disponible en: https://doi. org/10.2478/hukin-2018-0030

18. Mikić, M; Vučković, I; Karać, A; Belegišanin, B; Vukićević, V; Stojanović, $M$. Game-related statistics that discriminated winning and losing teams in NLB league. FU Phys Ed Sport. [Internet]. 2018 [Consultado 2020 Dic 16]; 16(2): 477-486. Disponible en: https://doi.org/10.22190/ FUPES180606042M

19. Ortega-Toro, E; Fernández-Verdejo, R; Ubal-Rodriguez, M; LorenzoCalvo, A; Sampaio, J. Indicadores de rendimiento defensivo en baloncesto en los ganadores y perdedores. Rev. int. cienc. deporte. [Internet]. 2010 [Consultado 2020 Dic 22]; 6(19): 100-11. Disponible en: doi:10.5332/ ricyde2010.01901

20. González-Ramirez, A; Fernández, G. Eficacia de los Sistemas Defensivos en los Playoff de la Liga Uruguaya de Básquetbol 2012-2013. Rev. Univ. Educ. fis. deport. 2015; 8(8): 23:30.

21. Dogan, I; Ersoz, Y. The important Game-Related Statistics for Quialifying Next Round in Euroleague. Monten. J. Sports Sci. Med. [Internet]. 
2019 [Consultado 2020 Dic 12]; 8(1): 43-50. Disponible en : 10.26773/ mjssm.190307 (doi.org)

22. García, J; Ibañez, JS; Martinez-De Santos, R; Leite, N; Sampaio, J. Identifying Basketball Performance Indicators in Regular Season and Playoff Games. JOHK [Internet]. 2013 [Consultado 2020 Dic 12]; 36(1): 161168. Disponible en: https://doi.org/10.2478/hukin-2013-0016

23. Mateus, N; Gonçalves, B; Abada, E; Leite, N; Gómez, MA; Smapaio, J. Exploring game performance in NBA playoffs. Kinesiology. [Internet]. 2018 [Consultado 2020 Dic 22]; 50(1): 89-96. Disponible en: https://doi. org/10.26582/k.50.1.7

24. García, J; Ibañez, SJ; Feu, S. Estadísticas de juego que discriminan las selecciones participantes en los Campeonatos del Mundo 2006 de baloncesto, en función del nivel del nivel y género de los equipos. Kronos. 2010; 9(17): 57-63.

25. Madarme, H. Age and sex differences in game-related statistics which discriminate winners from losers in elite basketball games. Motriz: rev. educ. fis. [Internet]. 2018 [Consultado 2020 Dic 22]; 24(1): e1018153. Disponible en: https://doi.org/10.1590/s1980-6574201800010001

26. Guimarães, E; Santos, A; Santos, E; Tavares, F; Janeira, MA. National Players vs. Foreign Players: what distinguishes their game performance? A study in the Portuguese Basketball League. Rev. Int. cienc. Deporte. [Internet]. 2018 [Consultado 2020 Dic 14]; 14(54): 374-381. Disponible en: https://doi.org/10.5232/ricyde2018.05407

27. Gasperi, L; Conte, D; Leicht, A; Gómez-Ruano, MA. Game Related Statistics Discriminate National and Foreign Players According to Playing Position and Team Ability in the Women's Basketball EuroLeague. Int. J. Environ. Res. Public Health. [Internet]. 2020 [Consultado 2020 Dic 14]; 17(15): 1-10. Disponible en: https://doi.org/10.3390/ijerph17155507

28. Manasses, I. A study of behavior of Romanian and foreign basketball players in the U-Mobitelco team and FIBA Eurochallenge Cup games. Palestrica of the Third Millennium Civilization \& Sport. 2015; 16(4): 347-350.

29. Sampaio, J; Ibañez, SJ; Gómez, MA; Lorenzo, A; Ortega, E. Game location influences basketball players' performance across playing positions. INT J Sport Psychol. 2013; 39(3): 205-216. 